\title{
3D printing of composite reflectors for enhanced light collection in scintillation detectors
}

This paper was downloaded from TechRxiv (https://www.techrxiv.org).

\section{LICENSE}

CC BY 4.0

SUBMISSION DATE / POSTED DATE

25-07-2021 / 26-07-2021

CITATION

Sokolov, Petr; Komissarenko, Dmitrii; Dosovitskiy, Georgy; Korzhik, Mikhail (2021): 3D printing of composite reflectors for enhanced light collection in scintillation detectors. TechRxiv. Preprint.

https://doi.org/10.36227/techrxiv.15049911.v1

DOI

10.36227/techrxiv.15049911.v1 


\title{
3D printing of composite reflectors for enhanced light collection in scintillation detectors
}

\author{
Petr S. Sokolov ${ }^{1,2}$ *, Dmitrii A. Komissarenko², Svetlana K. Belus², Georgy A. Dosovitskiy ${ }^{1,2}$, \\ Dmitry Yu. Kozlov ${ }^{3}$, Alexey E. Dosovitskiy ${ }^{4}$ and Mikhail V. Korzhik ${ }^{1,3}$ \\ 1. National Research Centre "Kurchatov Institute”, 123182 Moscow, Russia \\ 2. NRC "Kurchatov Institute" - IREA, 107076 Moscow, Russia \\ 3. Institute for Nuclear Problems of Belarus State University, 220030 Minsk, Belarus \\ 4. NeoChem JSC, Moscow, Russia
}

Keywords: 3D printing; Light yield; Pixel array; Reflection coating; Scintillator; Wrapping

\begin{abstract}
The present study deals with the fabrication of light-reflecting materials used in pixelated scintillator detectors. For the first time, the reflecting surfaces for pixels of different sizes (from 0.8 to $3.2 \mathrm{~mm}$ ) were obtained via a low-cost DLP 3D printing technique. The material for the reflectors was the new composite of transparent ultraviolet light-cured resin and $\mathrm{TiO}_{2}$ as a light-scattering filler. It was observed that $\mathrm{TiO}_{2}$ showed better performance compare to other pigments such as $\mathrm{BaSO}_{4}, \mathrm{hBN}$ or cubic zirconia. The object formation rate was about $1 \mathrm{~cm}$ per hour with the possibility to produce several parts simultaneously that simplifies the wrapping procedure. It was found that the regular grooves pattern of the fabricated parts (staircase effect) could increase a light collection from a scintillator. The reflective properties of such surfaces were comparable to conventional reflection coating (e.g., Teflon wrapping).
\end{abstract}

\section{Introduction}

One of the important parts of a scintillation detector is a reflector of a scintillation element. The reflecting coating greatly affects an amount of light collected from the used scintillator and, thus, it can significantly improve the final resolution of the detector. In common practice of scintillation measurements, a Teflon tape is considered as a standard wrapping [1, 2]. It allows to obtain good results in light collection, however as a delicate manual work, especially for small elements, this approach is time-consuming and quite often unreproducible. Moreover, it is rather inconvenient, when the scintillator pixel array has to be assembled. Few complex procedures have been developed to overcome this problem, although they are laborious and difficult to automate [3, 4]. A method of forming a coating directly on the surface of a scintillation element by means of its physicochemical treatment is also known [5]. Thus, it could be interesting to develop an alternative approach which can simplify this procedure.

\footnotetext{
* Corresponding authors. Email: sokolov.petr@ gmail.com (Dr. Sokolov P.S.) and george.dos@ gmail.com (Dr. Dosovitskiy G.A.); Tel: +7 495963 7350; Postal address: NRC "Kurchatov Institute" - IREA, Bogorodskiy val str. 3; Moscow, 107076, Russia.
} 
In our previous work, we have successfully printed the scintillators of YAG polycrystalline ceramic using a digital light processing (DLP) method [6]. The DLP is an additive manufacturing technique which allows to fabricate objects with complex shape, high dimensional resolution and fine surface finishing $[6,7,8]$. The method is based on the layer-by-layer solidification of a liquid photosensitive resin via UV-light exposure. The present work aims to explore the DLP 3D printing approach to the formation of reflectors for single pixels and pixel arrays, which could significantly simplify an assembling procedure. The influence of UV-light exposure parameters on the crucial characteristics of a 3D printing slurry, such as cure depth, will be discussed in details. Using scanning electron microscopy, optical and scintillation measurements, it was shown that the printed surfaces are suitable for application in scintillator detectors.

\section{Experimental details}

The reflectors were made from a composite, consisting of a transparent polymer, filled with white inorganic pigment. $\mathrm{TiO}_{2}$ with both anatase (Himmed, Russia) and rutile (Zecchi, Italy) crystal structures was used as main fillers due to its high reflective properties. The following compounds were also used as pigment fillers: $\mathrm{BaSO}_{4}$ mark $\mathrm{AB} 113830$ (Abcr GmbH, Germany), hBN (Plasmotherm, Russia) and cubic $\mathrm{ZrO}_{2}$ (mark 8YSZ-1000C-2h, NeoChem JSC, Russia) [9].

The phase purity of inorganic fillers was confirmed by Raman spectroscopy and X-ray powder diffraction. The Raman spectra were recorded on a Vertex 70 Spectrometer with RAM II FT modulus (Bruker, Germany). The powders' X-ray diffraction analysis was carried out in a Guinier G670 chamber (Huber, Germany) with monochromatic $\mathrm{CuK}_{\alpha 1}$ radiation. The crystal lattice parameters and the phase composition were estimated using a PowderCell 2.4 program. The crystallite sizes of $\mathrm{TiO}_{2}$ powders were calculated by the Williamson-Hall method. The powder particles' morphology was studied using a JSM 7100F scanning electron microscope (SEM) (Jeol, Japan). The particle-size distributions were measured by laser diffraction using a MasterSizer 2000 (Malvern instrument, UK). The specific surface area and pore volume of the pigment powders were carried out in a TriStar 3000 analyser (Micromeritics, USA) using a Brunauer-Emmett-Teller (BET) and Barrett-Joyner-Halenda (BJH) methods, respectively.

Commercially available resins PR48 and Clear-No UVB (Colorado Photopolymer Solution, USA) were used for the preparation of the printing slurries with pigment concentrations in a range of 5 - 63 wt.\%. PR48 resin is an open-source mixture of acrylate monomers and oligomers with the addition of a UV photoinitiator and a UV blocker, ensuring necessary combination of characteristics: low viscosity in the liquid form, good photopolymerisability, satisfactory strength and flexibility in the cured form [7]. Clear-No UVB resin has the same composition as PR48, but does not contain the UV blocker. In some cases, liquid UV photoinitiator TPO-L (CAS 84434-11-7) was added at 1.0 wt.\% into curable formulations. The preparation of homogeneous UV-curable suspension (slurry) contained several steps: the pigment sieving, drying and mixing with the resin using a SpeedMixer DAC 400.2 Vac-P (Hauschild, Germany) machine. Finally, the suspensions were treated in an ultrasonic bath and degassed under a vacuum of 10 mbar. The rheological behaviour of the suspensions was studied using a Physica MCR 52 rheometer (Anton Paar, Austria).

An open-source 3D printer Ember (Autodesk, USA) [7] was used in the present study to fabricate composite reflectors. Its core working principle is a layer-by-layer polymerisation of liquid monomers upon light irradiation through a bottom of a printing vat. The lateral resolution of the printer is $50 \mu \mathrm{m}$. Before the fabrication procedure, the cure depth of the slurries was determined via polymerisation of a thin layer of the suspension at different UV-exposure times via a digital light 
projector (wavelength $405 \mathrm{~nm}$, light intensity $20 \mathrm{~mW} / \mathrm{cm}^{2}$ ) of the same printer. The 3D CAD models were designed using open-source FreeCAD v0.17 software. Print Studio v1.6.5 (Autodesk, USA) was used for slicing and creating print files.

Images of the reflectors were taken on a SU1510 scanning electron microscope (Hitachi, Japan). The reflectance curves of the composite polymer materials were recorded on Specord 250 Plus spectrophotometer. $100 \mu \mathrm{m}$-thick films were used for the measurements. Reflectors with different geometries were printed, for pixels ranging from submillimeter sizes to several centimetres. Scintillation light yield for GAGG:Ce and BGO scintillators was measured to compare the performance of different reflectors.

\section{Results and discussion}

Titanium oxide occurs in two polymorphs at ambient condition - the stable rutile, and metastable anatase [10]. These polymorphs exhibit different physical properties and consequently may express different behaviour in 3D printing and composites. Rutile is denser than anatase (4.25 versus $3.89 \mathrm{~g} / \mathrm{cm}^{3}$ ), has a higher refractive index (2.79 versus 2.54 ) and has a lower optical bandgap (3.0 versus $3.2 \mathrm{eV}$ ) [9]. The average aggregate size of both $\mathrm{TiO}_{2}$ powders was smaller $1 \mu \mathrm{m}$ according to laser diffraction data (see Supplementary Information Fig. S1). The dominant $\mathrm{TiO}_{2}$ phase for pigment from Himmed is anatase (above $99 \%$ ) and for pigment from Zecchi is rutile (above $98 \%$ ) as demonstrated in Fig. S2 and S3. The lattice parameters were determined as $a=3.784(1) \AA$, $c=9.513(1) \AA$ for anatase and $a=4.595(1) \AA, c=2.959(1) \AA$ for rutile, which very close to the literature values [10]. A mean crystallite sizes from the X-ray data are 120 and $140 \mathrm{~nm}$ for anatase and rutile respectively. The BET specific surface area of the $\mathrm{TiO}_{2}$ powders are 9.8 and $16.7 \mathrm{~m}^{2} / \mathrm{g}$ for anatase and rutile respectively. The $\mathrm{BJH}$ pore volume of $\mathrm{TiO}_{2}$ powders are 0.036 and $0.049 \mathrm{~cm}^{3} / \mathrm{g}$ for anatase and rutile respectively. The SEM has shown that the shape of the particles was spherical and the particle size was about $100 \mathrm{~nm}$, which is consistent with the BET measurements and results of XRD analysis.

In DLP 3D printing of the resins filled with inorganic particles the control of rheological properties of a slurry is an essential parameter. The viscosity of both initial resins is similar and close to $0.3 \mathrm{~Pa}$ s. The viscosity of the suspensions with $\mathrm{TiO}_{2}$ powders increased with the increase of pigment loading, but remained below $1 \mathrm{~Pa}$ s (see Supplementary Information Fig. S4), which is suitable for DLP printing. All suspensions showed near-fluid behaviour, which was close to Newtonian flow and their rheological and curing characteristics were found to be appropriate at the maximum possible solid content of $30 \mathrm{wt} . \%$. The higher pigment loading in the slurries can be achieved with $\mathrm{BaSO}_{4}$ and $8 \mathrm{YSZ}$ powders. For instance, the maximum $\mathrm{BaSO}_{4}$ content was $63 \mathrm{wt} . \%$ with the viscosity of $4 \mathrm{~Pa} \mathrm{~s}$ and at $50 \mathrm{wt} . \%$ the viscosity of the slurry was less than $1 \mathrm{~Pa}$ s. However, visually polymerised films with $\mathrm{BaSO}_{4}$ were translucent due to a low difference between acrylate polymer $(n=1.45)$ and the salt $(n=1.63)$. In this regard, the $\mathrm{TiO}_{2}$ filled polymers were opaque and thus favourable for the fabrication of reflective surfaces.

Obtaining of reflecting surfaces by UV photopolymerisation method is a self-contradictory task. Only relatively thin layer can be cured in one step. The cure depth versus the energy dose for PR48 and ClearNo UVB based suspensions is presented in Figs. 1 and 2, respectively. In all cases, the thickness of the cured layer increases with the energy dose applied. For $\mathrm{TiO}_{2} / \mathrm{PR} 48$ suspensions the cure depth dropped with increase of dispersion concentration due to a light scattering. It is known that the introduction of particles with high refractive index in a photocurable media induces the light scattering, suppresses the light transmission and significantly reduces the penetration depth of UV 
light through the suspensions [11]. For example, pure PR48 resin has a layer thickness of $180 \mu \mathrm{m}$ at $150 \mathrm{~mJ} / \mathrm{cm}^{2}$, while the cure depth of slurry with $10 \mathrm{wt} . \%$ of $\mathrm{TiO}_{2}$ (in anatase modification) is $80 \mu \mathrm{m}$ at the same energy dose (Fig. 1). The Ember 3D printer is not able to cure rutile/PR48 suspension with loading above $3 \mathrm{wt}$ \% even at the excessive exposure times.

The cure depth of Clear-No UVB resin is much higher than that of PR48 resin, due to absence of UV blocker (Fig. 2). The use of Clear-No UVB can remarkably increase the pigment concentration in the suspensions compare to PR48 pigment filled resin. For instance, the layer thickness of ClearNo UV resin with $30 \mathrm{wt} . \%$ of anatase particles was $60 \mu \mathrm{m}$ at $120 \mathrm{~mJ} / \mathrm{cm}^{2}$, whereas suspension with UV-blocker was uncurable at the same conditions and solid content. At the same time, available layer' thickness for $10 \mathrm{wt} . \%$ loading of rutile is about $50 \mu \mathrm{m}$ (exposure time is more than $10 \mathrm{~s}$ ). Addition of $1 \mathrm{wt} . \%$ UV photoinitiator increases the available cure thickness from 50 to $65 \mu \mathrm{m}$ at an energy of about $100 \mathrm{~mJ} / \mathrm{cm}^{2}$ (curve 3 versus curve 4 in Fig. 2) and from 50 to $130 \mu \mathrm{m}$ at an energy of about 250 $\mathrm{mJ} / \mathrm{cm}^{2}$ (curve 5 versus curve 7 in Fig. 2). Thus, the UV blocker in the initial resin drastically decreases available pigment loading. Conversely, the introduction of additional UV photoinitiator into the mixture might be a good way to increase available cure depth and/or pigment loading.

The slurry with 63 wt. $\%$ of $\mathrm{BaSO}_{4}$ has a cure depth of $400 \mu \mathrm{m}$ at the energy dose of 100 $\mathrm{mJ} / \mathrm{cm}^{2}$. The lower pigment content led to a significant increase in the cure depth of the slurry and in such a way 3D printing of the composites can be carried out only with a layer thickness of 100-200 $\mu \mathrm{m}$. Consequently, it can reduce the time to fabricate the reflective coating, but it will increase to a roughness of the surface. The cure depth of the suspensions filled with $10 \mathrm{wt} . \%$ and $20 \mathrm{wt} . \%$ of hBN at the energy dose of $100 \mathrm{~mJ} / \mathrm{cm}^{2}$ was 320 and $150 \mu \mathrm{m}$, respectively. The cure depth of the $8 Y S Z$ slurry at a dose of $100 \mathrm{~mJ} / \mathrm{cm}^{2}$ was about $40 \mu \mathrm{m}$ for the composition with the highest solid content of $40 \mathrm{wt} . \%$. However, the reflective properties of the composite were noticeably worse than those of a Teflon. Thus, among the all the analysed pigments, only titanium oxide in the anatase modification allows to obtain a composite with good reflective properties.

It is important to note that using a more powerful light source, the higher pigment loading and subsequently better reflection properties can be achieved. In the previous works, the highly loaded $\mathrm{TiO}_{2}$ suspensions were used to fabricate photonic crystals of $5 \mu \mathrm{m}$ layer thickness via microstereolithography process with high energy doses of $1000 \mathrm{~mJ} / \mathrm{cm}^{2}$ exposed to a layer [11, 12]. At the same time, examples of the DLP and LCD processes with low concentrated $\mathrm{TiO}_{2}$ slurries are also well known $[13,14]$. Perhaps a top-down approach, which is widely used in large industrial 3D printers, will be more promising for thin wall structures and larger cells.

The choice of surface design remains an open question. It can be assumed that flexible tortuous (self-sustaining and at the same time macro-ordered) thin-walled structures can have better mechanical and reflective properties than extended planar and/or vertical surfaces. The choice of a more flexible or strength polymeric material (or the introduction of a plasticizer in the current formulation) may be a further direction of the work.

The reflectance curves of polymer/pigment compositions are shown in Fig. 3. The films have a good reflectance in the region above $400 \mathrm{~nm}$. The reflection significantly improves with increase of the pigments content. Composites, containing 25 or more wt.\% of the pigment, demonstrated the reflective properties competitive to Teflon tape in the spectral region of interest $-500-550 \mathrm{~nm}$ (curves $5 \& 6)$. The reflective properties of anatase- and rutile-based composites are similar at equal pigment loadings (see curves 2 and 3 in Fig. 3). The SEM study of the fracture surfaces of 3D printed reflectors demonstrated that the $\mathrm{TiO}_{2}$ particles were well dispersed in the polyacrylate matrix (see Fig. S5 in 
Supplementary Information). No large defects such as cracks, holes or delamination were observed on the printed parts according to the SEM analysis (Fig. 4).

The reflectors for $2 \times 2$ pixel array are shown in Fig. 5 and Fig. S6 as an example; the dimensions are: cell size $3.2 \times 3.2 \mathrm{~mm}$, inner wall thickness of $0.17 \mathrm{~mm}$ and cell height of $5.0 \mathrm{~mm}$. A surface texture (so-called stair steps) could be seen from both SEM and photos (Fig. 4, 5). Such patterns were occurred due to a layer-by-layer formation of an object during 3D printing. The direction of these "stairs" depends on the object's orientation during its formation (in other words, on 3D printing direction). According to our observations, this feature of the reflectors made with 3D printing improves a light collection.

The photopeak positions of a $662 \mathrm{keV}$ from ${ }^{137} \mathrm{Cs}$ are presented in Table 1 and Fig. S7. The height pulse spectra were recorded using GAGG:Ce and BGO pixels with dimensions of $3 \times 3 \times 5 \mathrm{~mm}^{3}$ covered by $3 \mathrm{D}$ printed reflector composites. All pixels of each crystal were made from the same ingot to evaluate light collection non-uniformity from cell to cell. As seen, the measured samples of reflecting surfaces give results similar to Teflon tape wrapping both with GAGG and BGO, enabling a close degree of the light collection with much simpler array assembly. Deviation of peak position from pixel to pixel was found to be less than $2 \%$. The printed reflectors demonstrate the light collection comparable to Teflon tape, despite slightly inferior reflectance for the pigment load, which was used. This might be due to a surface texture of the reflectors. These steps decrease the area of contact of the reflector with the pixel surface, thus making an internal reflection likelier. Notably, the $662 \mathrm{keV}$ peak position is slightly higher for the Type 2 reflector, indicating slightly better light collection. In this reflector, the steps' direction is along the pixel length. So, the addition effect could be proposed, comprising channelling of the light, which escaped the crystal, towards the photodetector.

\section{Conclusions}

For the first time, the DLP 3D printing technique was applied for the fabrication of reflective surfaces used in scintillation measurements. The compositions with photocuring characteristics appropriate for 3D printing were developed. It has been shown that various geometries with 0.17 $0.3 \mathrm{~mm}$ wall thicknesses, 0.8 - $3.2 \mathrm{~mm}$ cell width and up to $40 \mathrm{~mm}$ cell length can be made relatively easily using the cheap desktop DLP 3D printer. Reflecting properties of a mixture of commercial UVcurable resins PR48 or Clear-No UVB, and $\mathrm{TiO}_{2}$ pigment powders are comparable to Teflon, a conventional reflective material used for scintillator wrapping. The proposed 3D printing method allows quick fabrication of reflectors with different designs with high reproducibility, and the inherent surface texture of the 3D printed objects could be considered as photonic structures allowing better light transport along the scintillation elements.

\section{Acknowledgments}

The authors would like to thank D.E. Kuznetsova, P.V. Karpyuk, A.S. Nartov, P.A. Volkov for technical assistance during measurements. We are grateful to the NRC "Kurchatov Institute" IREA shared analytical facilities centre. Many thanks to Fomos Materials OJSC for collaboration, testing, and approbation of our 3D printed reflectors in his scintillation elements. This work was supported by a grant № 14.W03.31.0004 of Russian Federation Government. 


\section{References:}

1. M. Janecek, W.W. Moses, Optical reflectance measurements for commonly used reflectors, IEEE Trans. Nucl. Sci. 55 (2008) 2432-2437. DOI: 10.1109/TNS.2008.2001408

2. L. Stuhl, A. Krasznahorkay, M. Csatlós, A. Algora, J. Gulyás, G. Kalinka, Zs.I. Kertész, J. Timár, A newly developed wrapping method for scintillator detectors, J. Phys.: Conf. Ser. 665 (2016) 012050. DOI: 10.1088/1742-6596/665/1/012050

3. A. Tarancón, E. Marin, J. Tent, G. Rauret, J.F. Garcia, Evaluation of a reflective coating for an organic scintillation detector, Nucl. Instrum. Methods Phys. Res., Sect. A. 674 (2012) 92-98. DOI: 10.1016/j.nima.2012.01.048

4. C. Capoccia (2017.01), Calorimeter assembly and mechanics. Presented at PADME general meeting, Laboratori Nazionali di Frascati, INFN, Frascati, Rome, Italy

5. F. Meng, M. Koschan, M. Tyagi, C.L. Melcher, P. Cohen, A novel method to create an intrinsic reflective layer on a $\mathrm{Gd}_{3} \mathrm{Ga}_{3} \mathrm{Al}_{2} \mathrm{O}_{12}$ :Ce scintillation crystal, Nucl. Instrum. Methods Phys. Res., Sect. A. 763 (2014) 591-595. DOI: 10.1016/j.nima.2014.06.077

6. G.A. Dosovitskiy, P.V. Karpyuk, P.V. Evdokimov, D.E. Kuznetsova, V.A. Mechinsky, A.E. Borisevich, A.A. Fedorov, V.I. Putlayev, A.E. Dosovitskiy, M.V. Korjik, First 3D-printed complex inorganic polycrystalline scintillator, CrystEngComm 19 (2017) 42604264. DOI: 10.1039/C7CE00541E

7. B. Adzima. (03.2015), The Ember Printer: An Open Platform for Software, Hardware, and Materials Development. Presented at uv.eb. WEST Conference; Redondo Beach, CA. Available: $\quad$ https://www.radtech.org/images/presentations_papers/uvebwest2015/3 D_Printing/The_Ember_Printer-Adzima.pdf

8. D.A. Komissarenko, P.S. Sokolov, A.D. Evstigneeva, I.A. Shmeleva, A.E. Dosovitsky, Rheological and curing behavior of acrylate based suspensions for the DLP 3D printing of complex zirconia parts, Materials 11 (2018) 2350. DOI: 10.3390/ma11122350

9. P.S. Sokolov, P.V. Karpyuk, G.A. Dosovitskiy, P.A. Volkov, N.V. Lyskov, I.V. Slyusar, A.E. Dosovitskiy, Stabilized zirconia-based nanostructured powders for solid-oxide fuel cells, Russ. J. Electrochem. 54 (2018) 464-470. DOI: 10.1134/S1023193518060162

10. D.A.H. Hanaor, C.C. Sorrell, Review of the anatase to rutile phase transformation, J. Mater. Sci. 46 (2011) 855-874. DOI: 10.1007/s10853-010-5113-0

11. W. Chen. S. Kirihara, Y. Miyamoto, Fabrication of three - dimensional micro photonic crystals of resin - incorporating $\mathrm{TiO}_{2}$ particles and their terahertz wave properties, J. Am. Ceram. Soc. 90 (2007) 92-96. DOI: 10.1111/j.1551-2916.2006.01377.x

12. S. Kirihara, K. Nonaka, S. Kisanuki, H, Nozakim K. Sakaguchi, Ultraviolet laser lithography of titania photonic crystals for terahertz-wave modulation, Materials 11 (2018) 835. DOI: $10.3390 / \mathrm{ma1} 1050835$

13. J. Guo, Y. Zeng, P. Li, J. Chen, Fine lattice structural titanium dioxide ceramic produced by DLP 3D printing, Ceram. Int. 45 (2019) 23007-23012. DOI: 10.1016/j.ceramint.2019.07.346 
14. A. Malas, D. Isakov, K. Couling, G.J. Gibbons, Fabrication of high permittivity resin composite for vat photopolymerization 3D printing: morphology, thermal, dynamic mechanical and dielectric properties, Materials 12 (2018) 3818. DOI: 10.3390/ma12233818

Author's ORCID IDs:

Dr. Sokolov P.S. https://orcid.org/0000-0003-3516-3953

Dr. Komissarenko D.A. https://orcid.org/0000-0002-1575-9357

Dr. Dosovitskiy G.A. https://orcid.org/0000-0001-8219-3624

Dr. Korzhik M.V. https://orcid.org/0000-0001-8334-1502

\section{Highlights}

A new light reflecting material comprising $\mathrm{TiO}_{2}$ and a binder resin have been studied. Reflectors with complex shapes were formed via a low-cost DLP 3D printing technique. Reflecting properties of the composite are comparable to standard Teflon wrapping. The reflectors can be successfully used in scintillator arrays assembling.

\section{CRediT author statement}

Sokolov P.S.: Conceptualization, Visualization; Komissarenko D.A.: Writing - Original Draft; Belus S.K.: Investigation; Dosovitskiy G.A.: Conceptualization, Writing - Review \& Editing, Project administration; Kozlov D.Yu.: Investigation; Dosovitskiy A.E.: Project administration, Resources; Korzhik M.V.: Funding acquisition, Supervision.

\section{Declaration of interests}

The authors declare that they have no known competing financial interests or personal relationships that could have appeared to influence the work reported in this paper. 
Table 1. Comparison with teflon for GAGG:Ce and BGO pixels.

\begin{tabular}{|c|c|c|c|}
\hline $\begin{array}{l}\text { Sidewall reflecting } \\
\text { surface }\end{array}$ & $\begin{array}{l}\text { End-face reflecting } \\
\text { surface }\end{array}$ & \multicolumn{2}{|c|}{$\begin{array}{c}662 \mathrm{keV} \text { photopeak position (in channels) } \\
\text { GAGG:Ce and BGO }\end{array}$} \\
\hline \multicolumn{2}{|c|}{ Teflon tape, 3 layers } & 600 & 220 \\
\hline Type 1 & PET film Lumirror & 592 & 217 \\
\hline Type 1 & Type 1 & 594 & 229 \\
\hline Type 2 & Type 2 & 607 & - \\
\hline Type 2 & Type 1 & $602-604$ & - \\
\hline
\end{tabular}




\section{Figures}

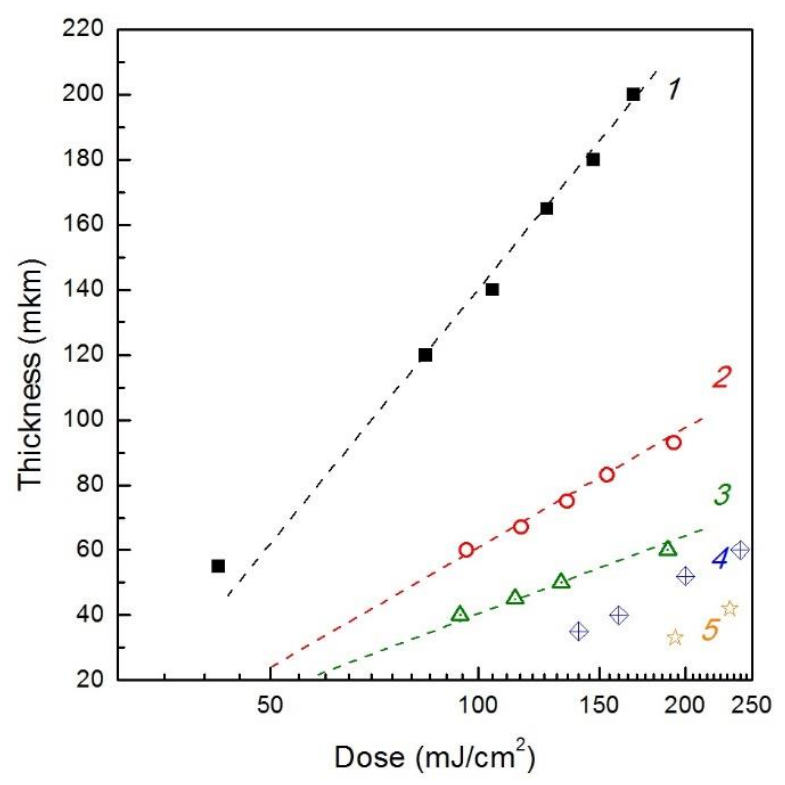

Figure 1. Layer thickness of the suspensions anatase $\mathrm{TiO}_{2} / \mathrm{PR} 48$ as a function energy dose applied (1) pure PR48; (2) 5 wt.\% $\mathrm{TiO}_{2}$; (3) 10 wt.\% $\mathrm{TiO}_{2}$; (4) 15 wt.\% $\mathrm{TiO}_{2}$ and (5) 20 wt.\% $\mathrm{TiO}_{2}$.

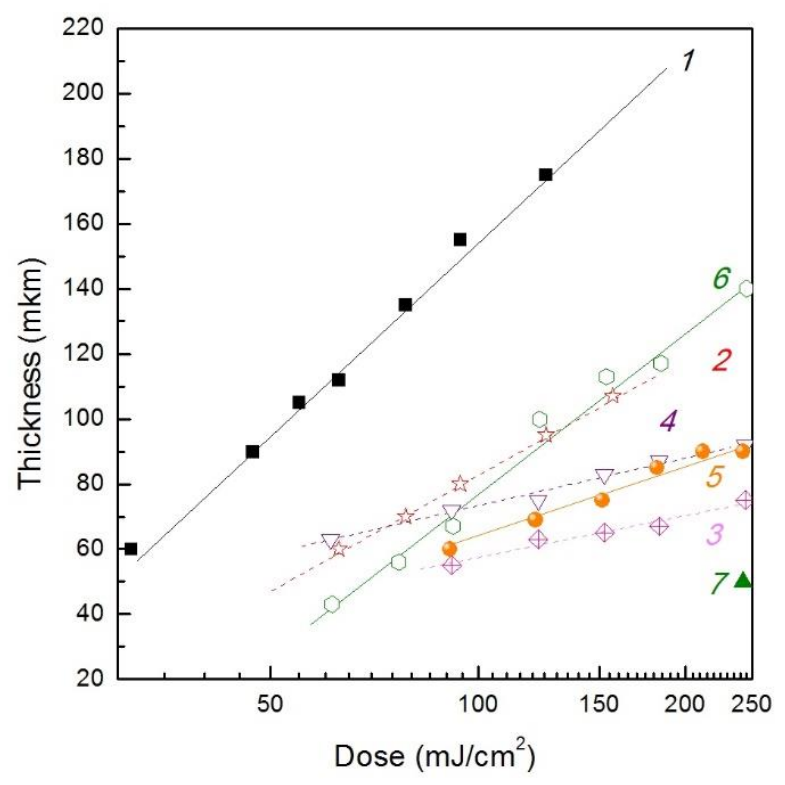

Figure 2. Layer thickness of the suspensions $\mathrm{TiO}_{2} / \mathrm{Clear}-\mathrm{No}$ UVB as a function energy dose applied (1) $10 \mathrm{wt} . \%$ anatase; (2) $20 \mathrm{wt} . \%$ anatase; (3) $30 \mathrm{wt} . \%$ anatase; (4) $30 \mathrm{wt} . \%$ anatase $+1 \mathrm{wt} . \%$ TPOL; (5) 10 wt.\% rutile + 1 wt.\% TPO-L; (6) 5 wt.\% rutile; (7) 10 wt.\% rutile. 


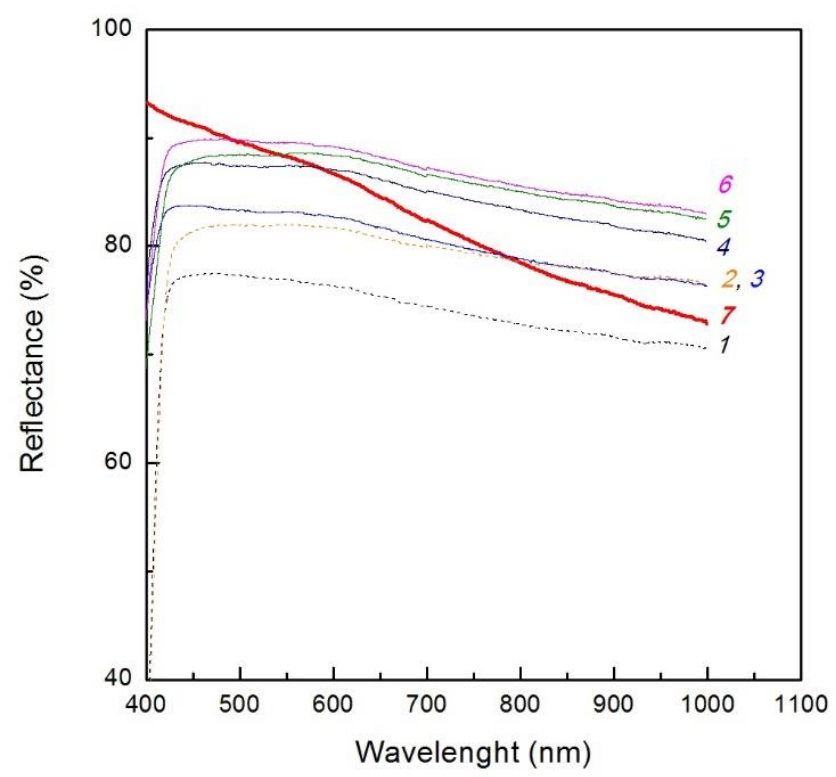

Figure 3. Reflecting of polymer/pigment compositions (1) 5 wt.\% rutile; (2) 10 wt.\% rutile; (3) 10 wt.\% anatase; (4) $20 \mathrm{wt} . \%$ anatase; (5) $25 \mathrm{wt} . \%$ anatase; (6) $30 \mathrm{wt} . \%$ anatase and (7) Teflon.

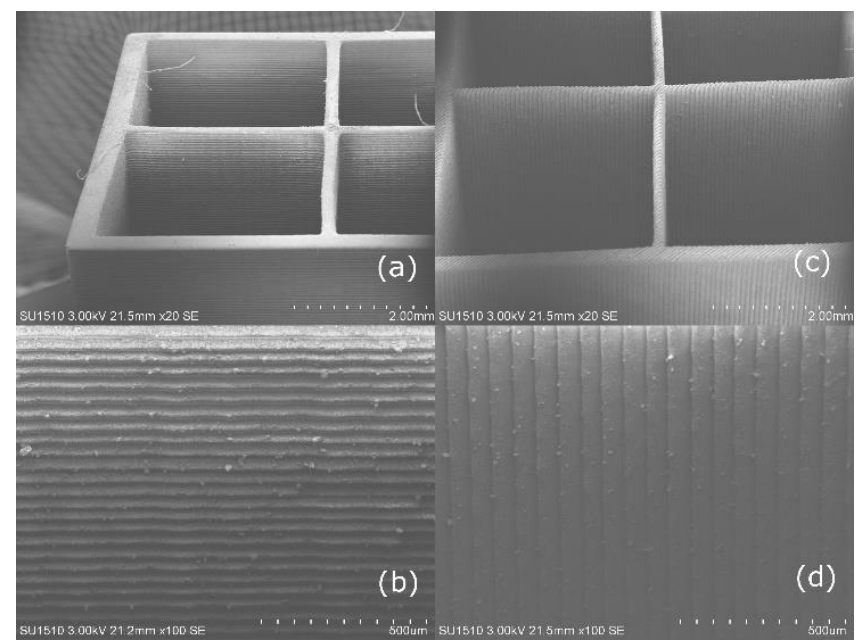

Figure 4. SEM images of 3D printed reflectors $10 \mathrm{wt} . \% \mathrm{TiO}_{2} / \mathrm{PR} 48,(\mathrm{a}, \mathrm{b})$ type 1 and (c, d) type 2. 


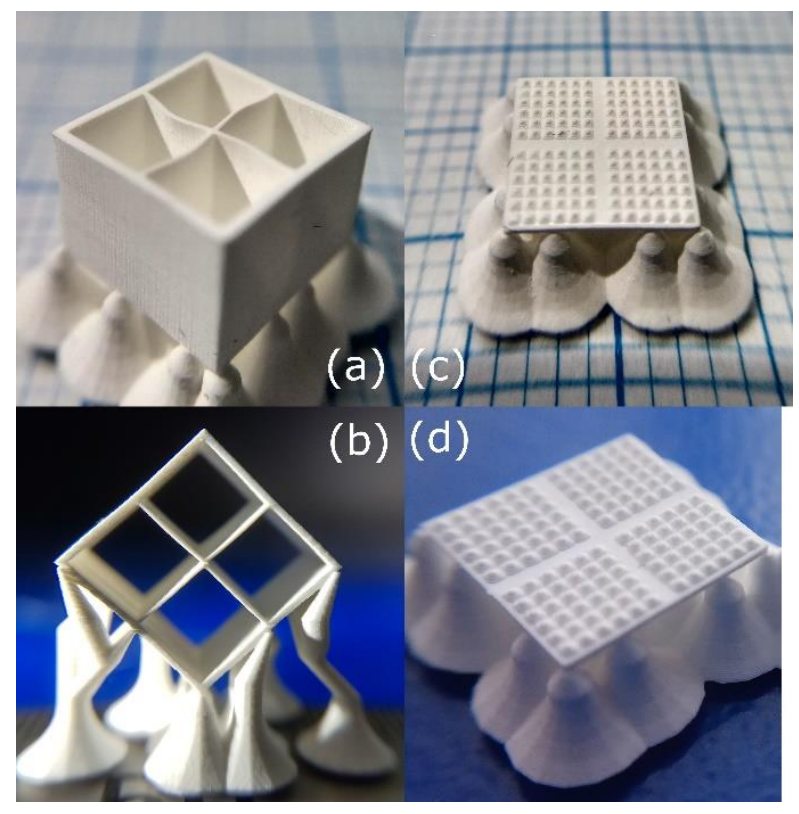

Figure 5. A photo of 3D printed reflectors $10 \mathrm{wt} . \% \mathrm{TiO}_{2} / \mathrm{PR} 48$, sidewall reflecting surfaces $(\mathrm{a}, \mathrm{b})$ type 1 and type 2; end-face reflecting surfaces (c, d) type 1 and type 2 . 


\section{Supplementary Information for 3D printing of composite reflectors for enhanced light collection in scintillation detectors}

Petr S. Sokolov, Dmitrii A. Komissarenko, Svetlana K. Belus, Georgy A. Dosovitskiy,

Dmitry Yu. Kozlov, Alexey E. Dosovitskiy and Mikhail V. Korzhik

This PDF file includes:

Figures S1, S2, S3, S4, S5, S6 and S7.

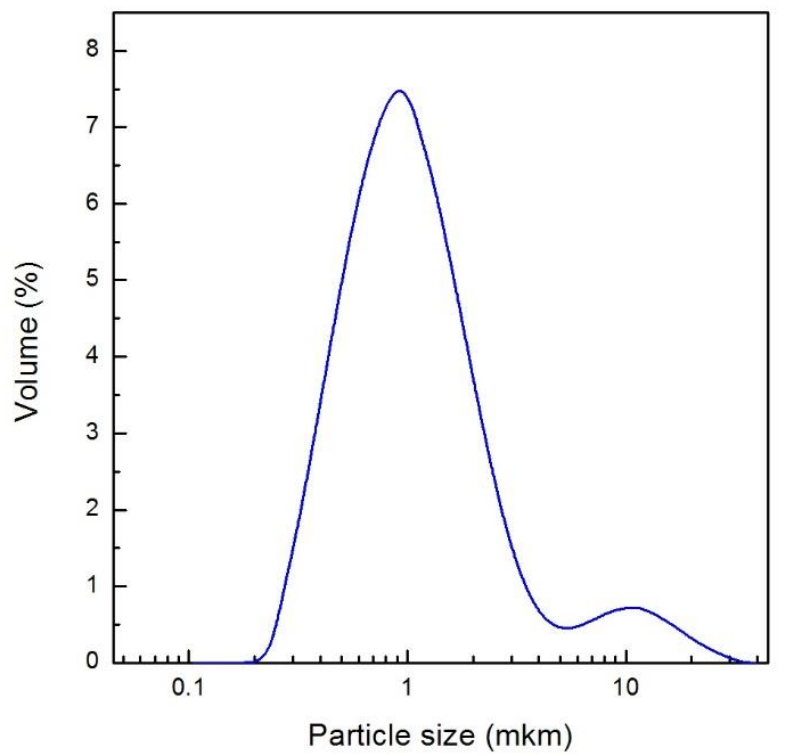

(a)

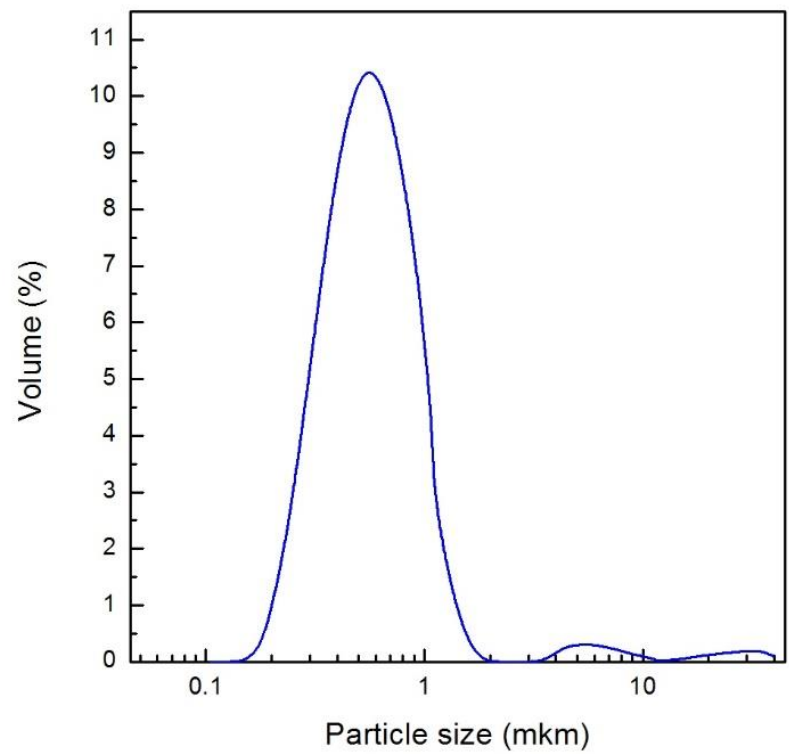

(b)

Fig. S1. Particle size distributions of $\mathrm{TiO}_{2}$ powders (a) anatase; (b) rutile.

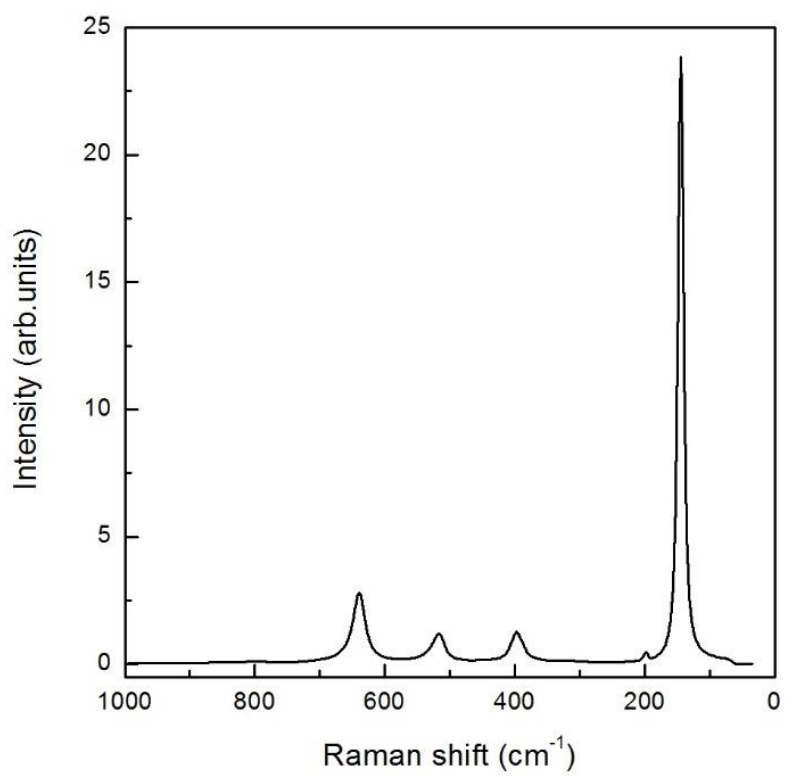

(a)

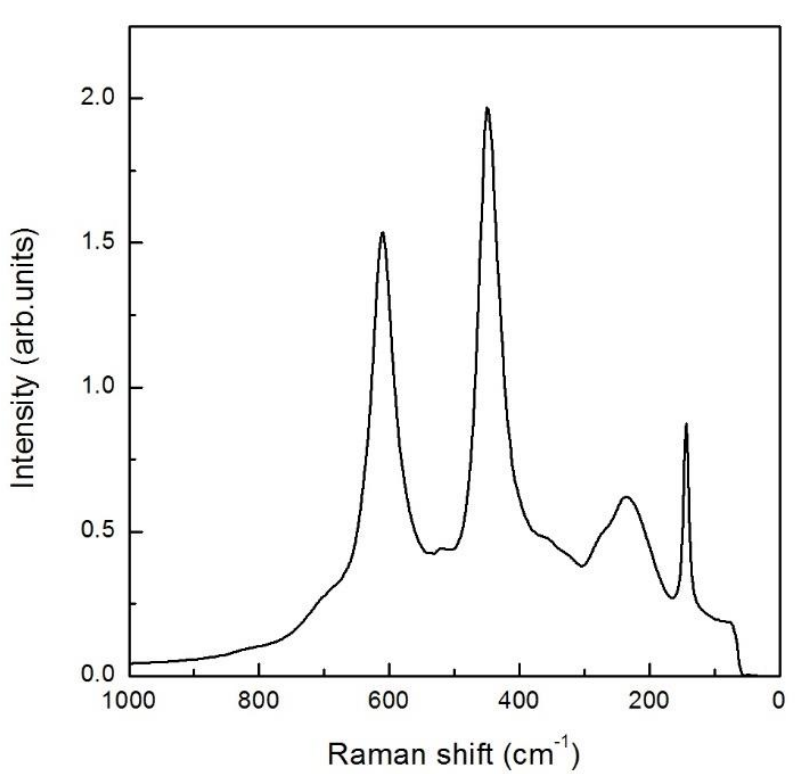

(b)

Fig. S2. Raman spectra of $\mathrm{TiO}_{2}$ powders (a) anatase; (b) rutile. 


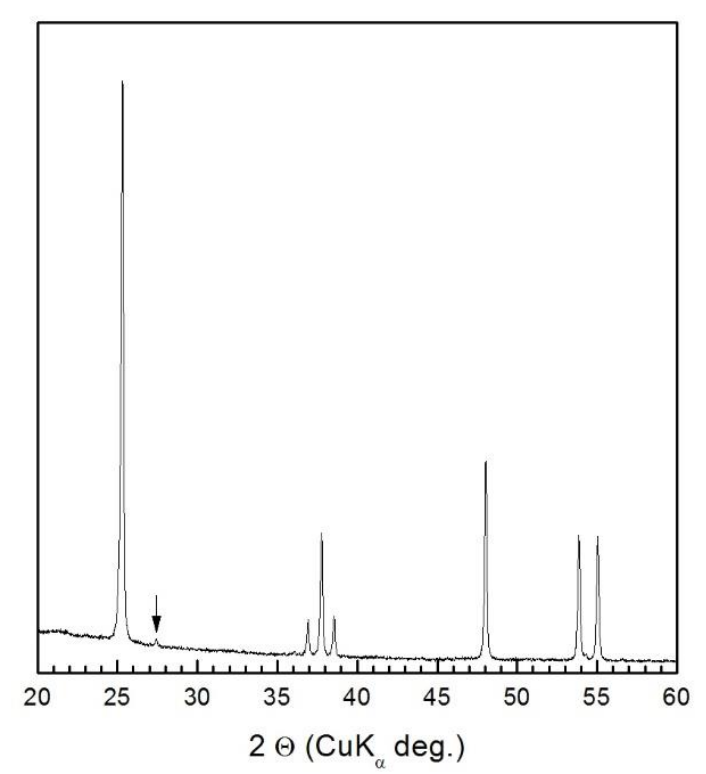

(a)

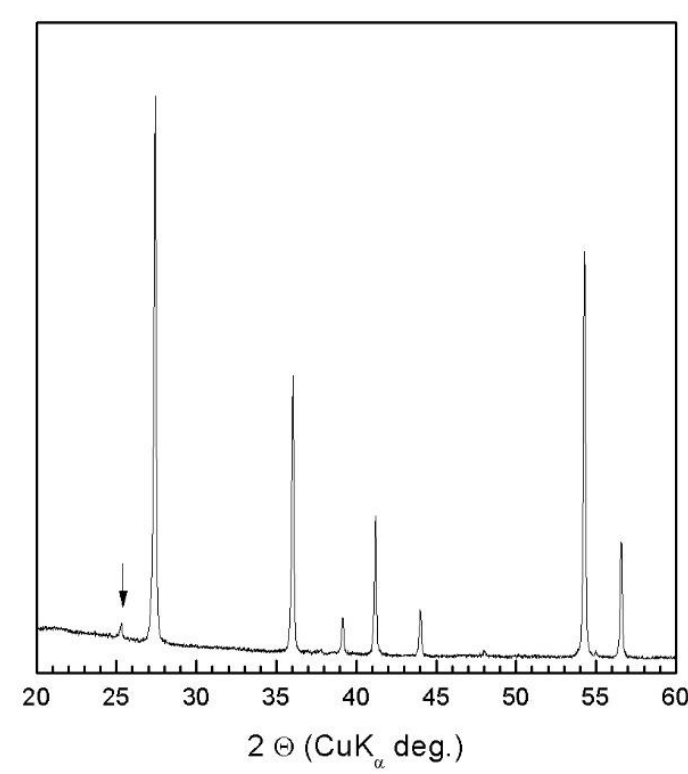

(b)

Fig. S3. Diffraction patterns of $\mathrm{TiO}_{2}$ powders

(a) main phase is anatase, the arrow shows the location of the 110 diffraction line of rutile;

(b) main phase is rutile, the arrow shows the location of the 101 diffraction line of anatase.

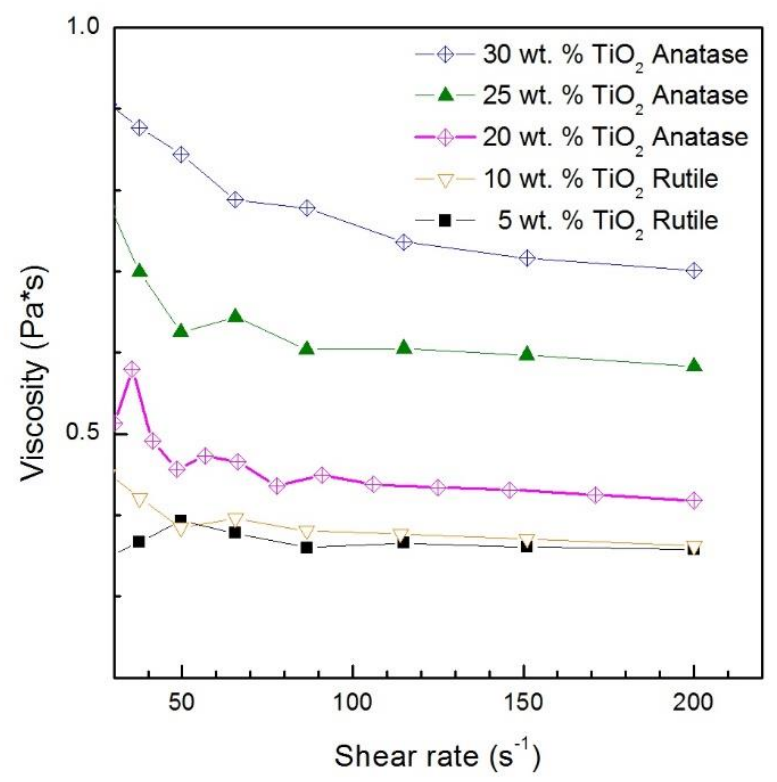

Fig. S4. Viscosity of different suspensions with Clear-No UVB resin. 


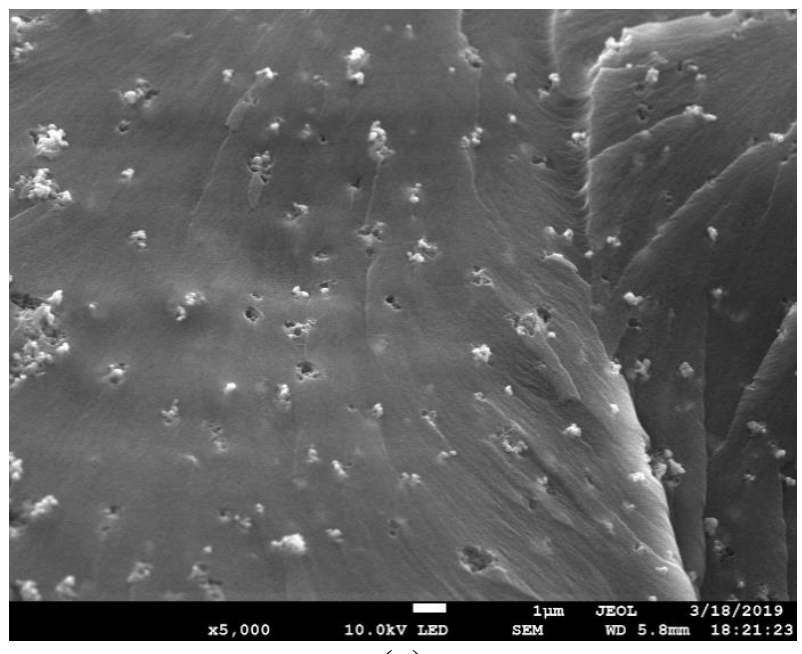

(a)

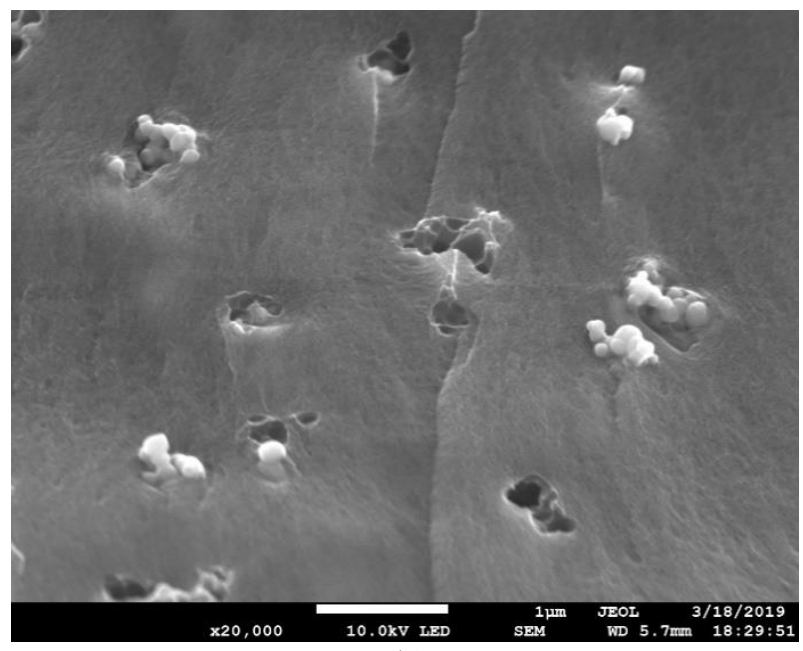

(b)

Fig. S5. The SEM images of the fracture surfaces of the 3D printed reflectors $10 \mathrm{wt} . \% \mathrm{TiO}_{2} / \mathrm{PR}_{4}$ (a) $5 \mathrm{kx}$; (b) $20 \mathrm{kx}$.
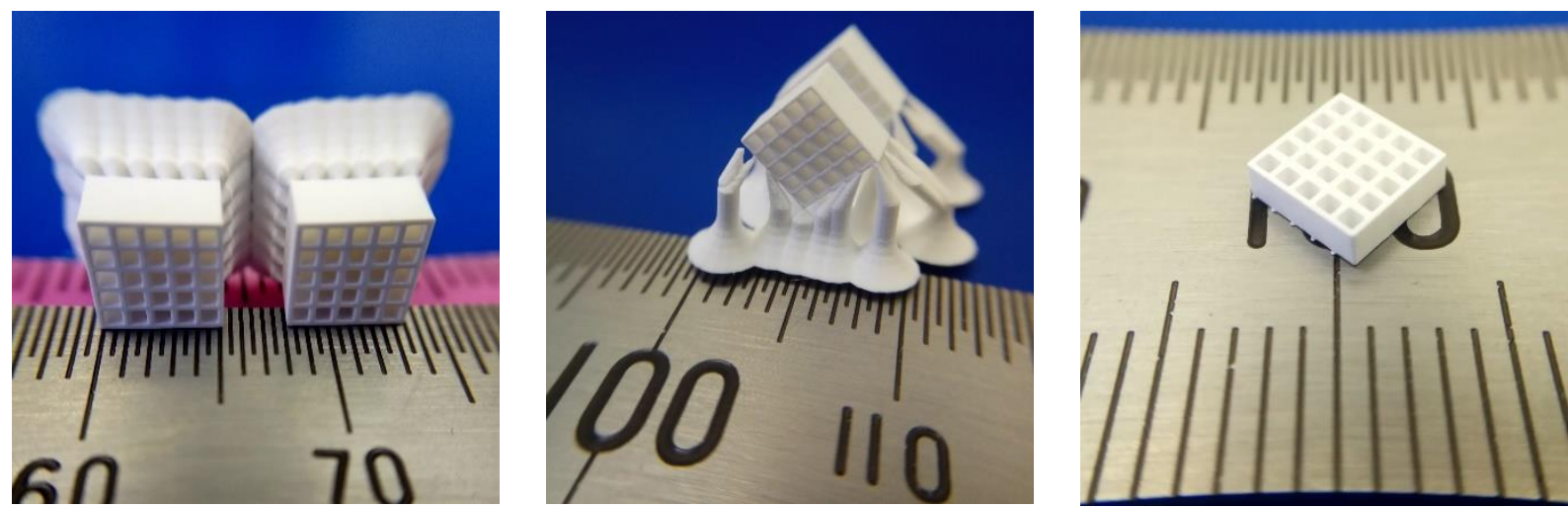

Fig. S6. Some more examples of geometry of 3D printed reflectors (30 wt.\% $\mathrm{TiO}_{2} /$ Clear-No UVB).

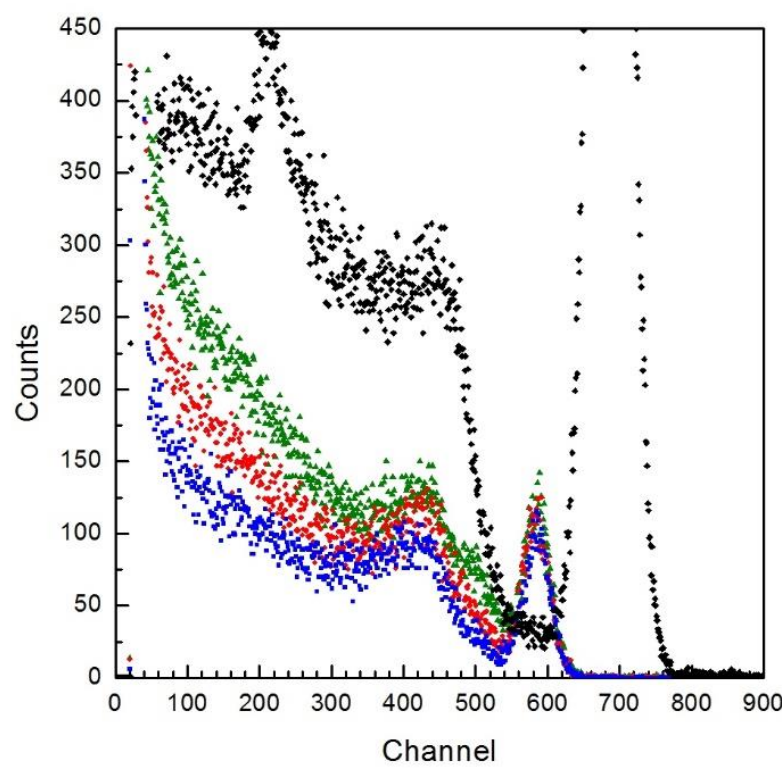

(a)

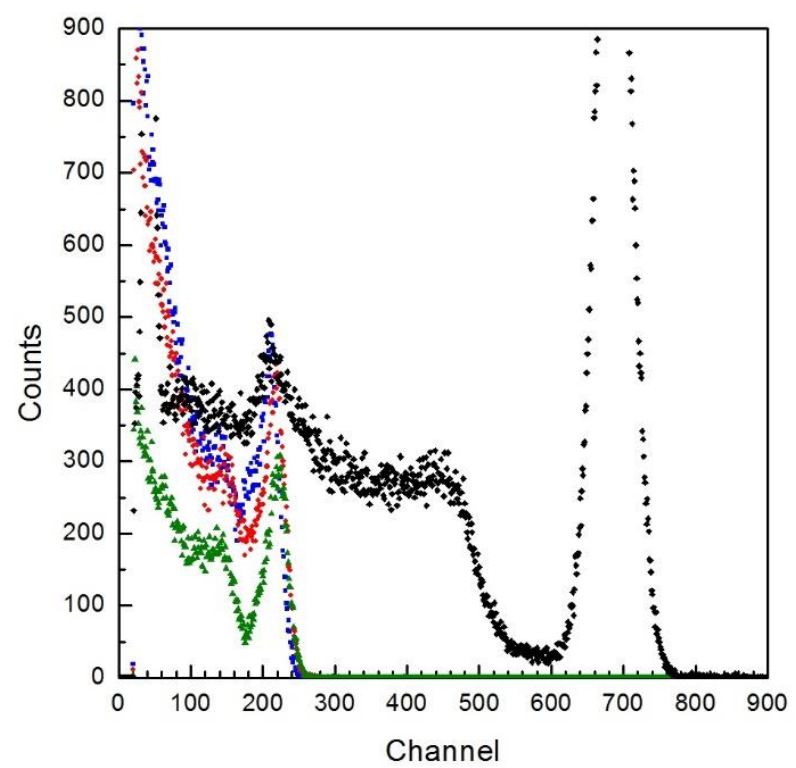

(b)

Fig. S7. (a) Pulse height spectra registered with GAGG pixels under $662 \mathrm{keV}{ }^{137} \mathrm{Cs}$ source in three neighboring cells of the 3D printed form. CsI:Tl is given as a benchmark (black dots); (b) Pulse height spectra registered with BGO pixels under $662 \mathrm{keV}{ }^{137} \mathrm{Cs}$ source in three neighboring cells of the 3D printed form. 